\title{
Curvature bound from gravitational catalysis in thermal backgrounds
}

\author{
Holger Gies $\circledast^{1,2, *}$ and Abdol Sabor Salek $\oplus^{1, \dagger}$ \\ ${ }^{1}$ Theoretisch-Physikalisches Institut, Abbe Center of Photonics, Friedrich Schiller University Jena, \\ Max Wien Platz, 1, 07743 Jena, Germany \\ ${ }^{2}$ Helmholtz-Institut Jena, Fröbelstieg 3, D-07743 Jena, Germany
}

(Received 17 March 2021; accepted 3 June 2021; published 28 June 2021)

\begin{abstract}
We investigate the phenomenon of gravitational catalysis, i.e., curvature-induced chiral symmetry breaking and fermion mass generation, at finite temperature. Using a scale-dependent analysis, we derive a thermal bound on the curvature of local patches of spacetime. This bound quantifies regions in parameter space that remain unaffected by gravitational catalysis and thus are compatible with the existence of light fermions as observed in nature. While finite temperature generically relaxes the curvature bound, we observe a comparatively strong dependence of the phenomenon on the details of the curvature. Our bound can be applied to scenarios of quantum gravity, as any realistic candidate has to accommodate a sufficient number of light fermions. We argue that our bound therefore represents a test for quantum-gravity scenarios: A suitably averaged spacetime in the (trans-)Planckian regime that satisfies our curvature bound does not induce correspondingly large Planckian fermion masses by gravitational catalysis. The temperature dependence derived in this work facilitates to follow the fate of gravitational catalysis during the thermal history of the (quantum) Universe. In an application to the asymptotic-safety scenario of quantum gravity, our bound translates into a temperature-dependent upper bound on the number of fermion flavors.
\end{abstract}

DOI: $10.1103 /$ PhysRevD.103.125027

\section{INTRODUCTION}

Chiral symmetry breaking and fermion mass generation is a central feature of interacting fermions relevant for both the Higgs sector of the Standard Model as well as QCD shaping many properties of matter in the Universe. Whereas the long-range limit of gravity in the form of Einstein's general relativity is too weakly interacting to affect the status of chiral symmetry, gravity is expected to become more strongly interacting at or above the Planck scale. Whether or not gravity or its quantized form may exert a strong influence on the chiral features of fermions deserves to be studied. In fact, such an influence may even be used as an observational probe for scenarios of quantum gravity: As suggested in [1], viable scenarios of quantum gravity need to be compatible with the existence of light fermion as observed in nature-a requirement that has the potential to impose constraints or even rule out certain scenarios of quantum gravity.

\footnotetext{
*holger.gies@uni-jena.de

†abdol.sabor.salek@uni-jena.de
}

Published by the American Physical Society under the terms of the Creative Commons Attribution 4.0 International license. Further distribution of this work must maintain attribution to the author(s) and the published article's title, journal citation, and DOI. Funded by SCOAP ${ }^{3}$.
It is reassuring to see that quantum fluctuations of the metric do not support the same kind of chiral symmetrybreaking mechanism as is triggered by spin-one gauge fields or Yukawa interactions with scalars [1-9]. For both latter cases, the gauge or Yukawa couplings simply have to increase beyond a certain threshold which renders chiral symmetry breaking in these scenarios a rather universal strong-coupling feature. This is not so in metric quantum gravity.

By contrast, gravity offers further mechanisms to trigger fermion mass generation which are generic to gravity in the sense that they proceed via the structure of spacetime itself. The most widely studied mechanism occurs on negatively curved spacetimes and can be summarized by gravitational catalysis [10]. It appears in a large variety of fermionic models [11-27], as it derives from a mechanism of dimensional reduction of the spectrum of the Dirac operator on hyperbolic spacetimes [28] (on positively curved spacetimes, curvature effects can still exert an influence on the fermion mass formation in combination with magnetic catalysis $[29,30])$. Another mechanism has recently been suggested and worked out in [31]: In quantum-gravity scenarios allowing for topology fluctuations, gravitational instantons can contribute to anomalous chiral symmetry breaking and thereby generate fermion masses potentially in conflict with observation. In combination with Abelian gauge interactions, gravity can trigger also conventional symmetry-breaking mechanisms, as demonstrated in [32]. 
In the present work, we further explore pure gravitational catalysis specifically by including the effects of finite temperature. Following an earlier zero-temperature analysis $[33,34]$, we study the phenomenon using a renormalizationgroup (RG) inspired scale-dependent approach. The advantage is that we can monitor the RG relevance of chiral interactions in this way. In fact, gravitational catalysis can be connected with four-fermion operators becoming RG relevant driving the symmetry-breaking interactions to criticality [35]. This makes the analysis of gravitational catalysis in the context of quantum-gravity scenarios more subtle: It is not sufficient to check whether the long-range curvature of spacetime is compatible with the existence of light fermions (which obviously is the case). Moreover, the influence of spacetime curvature on the symmetry-breaking operators has to be checked during the whole course of the RG flow, specifically in the Planckian regime and beyond. Provided a notion of curvature exists in that regime, gravitational catalysis could be active and drive the symmetry-breaking operators beyond criticality. This would result in correspondingly heavy fermions removing light fermions from the observable long-range spectrum. The precise connection between the curvature and the induced value of the fermion mass depends on the details of the induced fermion self-interactions; see, e.g., Ref. [35] for an explicit analysis. However, the scale for the induced masses is essentially set by the scale at which the symmetry-breaking operators become critical which in a quantum gravitational context would be clearly linked to the Planck scale.

This mechanism has been explored in [33] which lead to the notion of curvature bounds: In order to guarantee that a given quantum-gravity scenario is not affected by the problem of gravitational catalysis, the averaged curvature of a local patch of spacetime should not exceed a certain bound. So far, these bounds have been derived for Riemannian hyperbolic spacetimes such as $\mathbb{\boxplus}^{D}$ in general spacetime dimensions $D$; cf. [33].

In the present work, we generalize the analysis to $\mathbb{R} \otimes \mathbb{M}^{D-1}$ or $S^{1} \otimes \mathbb{M}^{D-1}$. The purpose is twofold: First, this provides further information about the concrete dependence of the mechanism on the details of the averaged spacetime structure.

Second, this allows one to monitor the influence of finite temperature on the mechanism. The latter is particularly relevant for studying the influence of gravitational catalysis in the course of the cosmological evolution. Indeed, our results provide evidence for a comparatively strong dependence of gravitational catalysis on the details of the background. At the same time, finite-temperature effects can significantly relax the curvature bounds-in line with the expectation that thermal fluctuations drive the system toward the disordered symmetric phase.

Our paper is organized as follows: Sec. II lays out the general framework of our study in terms of a generic chiral fermion theory in curved spacetime, which we analyze in a local mean-field RG approach. The essential technical ingredient for taking the curved as well as thermal background into account, namely the heat kernel, is briefly described in Sec. III. The curvature bounds for gravitational catalysis in a purely spatially hyperbolic spacetime with and without finite temperature are derived in Sec. IV. As an illustration, we apply these bounds from gravitational catalysis to the asymptotic-safety scenario for quantum gravity in Sec. V. In this application, our curvature bounds translate into an upper bound for the number of fermion degrees of freedom and zero temperature, and a combination of a bound on the fermion number and the temperature if considered within the context of a thermal history of the Universe. We conclude in Sec. VI.

\section{CHIRAL CHANNEL AND EFFECTIVE POTENTIAL}

In an RG picture, catalysis of chiral symmetry is triggered by four-fermion operators becoming RG relevant [35]. Considering $N_{\mathrm{f}}$ fermion flavors, we study the RG behavior of four-fermion operators with maximal chiral $U\left(N_{\mathrm{f}}\right)_{\mathrm{R}} \times U\left(N_{\mathrm{f}}\right)_{\mathrm{L}}$ symmetry as an example.

Operators with a lower degree of symmetry can be studied analogously. We focus on the so-called $(V)+(A)$ channel,

$$
S_{\mathrm{int}} \sim \int_{x}\left[\left(\bar{\psi}^{a} \gamma_{\mu} \psi^{a}\right)^{2}+\left(\bar{\psi}^{a} \gamma_{\mu} i \gamma_{5} \psi^{a}\right)^{2}\right],
$$

which is one out of the two Fierz-independent local interaction terms of maximal symmetry [36]. It is Fierz equivalent to the scalar-pseudoscalar channel of the Nambu-Jona-Lasinio (NJL) model which, using the projectors

$P_{\mathrm{L}}=\frac{\mathbb{1}-\gamma_{5}}{2}, \quad P_{\mathrm{R}}=\frac{\mathbb{1}+\gamma_{5}}{2}, \quad \mathbb{1}=P_{\mathrm{L}}+P_{\mathrm{R}}$

onto left and right chiral components, can be rearranged as

$$
S_{\text {int }}[\bar{\psi}, \psi]=-2 \int_{x} \bar{\lambda}\left(\bar{\psi}^{a} P_{\mathrm{R}} \psi^{b}\right)\left(\bar{\psi}^{b} P_{\mathrm{L}} \psi^{a}\right) .
$$

Here we have introduced a (dimensionful) coupling constant $\bar{\lambda}$ parameterizing the strength of the chiral interaction. In the NJL model, this coupling is tuned beyond a critical value $\bar{\lambda}>\bar{\lambda}_{\text {cr }}$ triggering chiral symmetry breaking in terms of initial conditions. Incidentally, a thermal environmentbreaking spacetime symmetries explicitly-allows for further sets of Fierz inequivalent interactions where spatial and temporal components of vector-type channels are treated independently [37-39]. In the following, we ignore this potential splitting and concentrate on the NJL channel. Here, we always assume the initial condition to be 
subcritical such that this operator does not generate fermion masses on its own.

Introducing a nondynamical Hubbard-Stratonovich field $\phi$, the chiral channel can be rewritten in terms of a local Yukawa interaction:

$$
\mathcal{L}_{\text {int }}[\phi, \bar{\psi}, \psi]=\bar{\psi}^{a}\left[P_{\mathrm{L}}\left(\phi^{\dagger}\right)_{a b}+P_{\mathrm{R}} \phi_{a b}\right] \psi^{b}+\frac{1}{2 \bar{\lambda}} \operatorname{tr}\left(\phi^{\dagger} \phi\right) .
$$

The equivalence between Eqs. (3) and (4) becomes obvious with the aid of the equation of motion for the chiral matrix field,

$$
\begin{aligned}
\phi_{a b} & =-2 \bar{\lambda} \bar{\psi}^{b} P_{\mathrm{L}} \psi^{a}, \\
\left(\phi^{\dagger}\right)_{a b} & =-2 \bar{\lambda} \bar{\psi}^{b} P_{\mathrm{R}} \psi^{a} .
\end{aligned}
$$

This scalar field, in fact, serves as an order parameter for the status of chiral symmetry. E.g., assuming a diagonalizable expectation value in flavor space, $\phi_{a b}=\phi_{0} \delta_{a b}$ with $\phi_{0}>0$ being homogeneous in spacetime, the chiral group breaks to a residual vector symmetry similar to QCD-like theories, and all fermions acquire masses of order $\phi_{0}$. Including a fermion kinetic term, the action reads

$$
S\left[\phi_{0}, \bar{\psi}, \psi\right]=\int_{x}\left\{\bar{\psi}\left(\not \nabla+\phi_{0}\right) \psi+\frac{1}{2 \bar{\lambda}} N_{\mathrm{f}}\left(\phi_{0}\right)^{2}\right\} .
$$

Our focus on a homogeneous condensate field $\phi_{0}$ may preclude a study of inhomogeneous condensates for which examples are known that yield a deeper global minimum of the effective potential (or free energy). If such a case occurred for gravitational catalysis, the bounds derived below would even be strengthened. Furthermore, we confine ourselves to integrating out the fermion degrees of freedom and neglect order parameter fluctuations in the following. In this way, we obtain a mean-field expression for the effective potential of the order parameter

$$
\begin{aligned}
\tilde{U}\left(\phi_{0}\right) & =\frac{N_{\mathrm{f}}}{2 \bar{\lambda}}\left(\phi_{0}\right)^{2}-N_{\mathrm{f}} \log \operatorname{Det}_{x}\left(\not \mathbf{D}+\phi_{0}\right) \\
& =\frac{N_{\mathrm{f}}}{2 \bar{\lambda}}\left(\phi_{0}\right)^{2}-\frac{N_{\mathrm{f}}}{2} \operatorname{Tr}_{x} \log \left(-\not \not^{2}+\phi_{0}^{2}\right),
\end{aligned}
$$

where we have used the $\gamma_{5}$-Hermiticity of the covariant Dirac operator in the last step. This mean-field approximation becomes exact in the limit of large fermion flavors $N_{\mathrm{f}} \rightarrow \infty$. With an emphasis on the Standard Model and its extensions in the following, for which $N_{\mathrm{f}} \geq 22.5$, we expect the mean-field level to be sufficiently accurate for our purposes. It is convenient to introduce the FockSchwinger proper-time representation,

$$
\tilde{U}\left(\phi_{0}\right)=\frac{N_{\mathrm{f}}}{2 \bar{\lambda}}\left(\phi_{0}\right)^{2}+\frac{N_{\mathrm{f}}}{2} \int_{0}^{\infty} \frac{d s}{s} \mathrm{e}^{-\phi_{0}^{2} s} \operatorname{Tr}_{x} \mathrm{e}^{\not{ }^{2} s},
$$

in order to arrive at the heat-kernel trace for the present differential operator of interest:

$$
\operatorname{Tr}_{x} \mathrm{e}^{\not^{2} s}=\operatorname{Tr}_{x} K\left(x, x^{\prime} ; s\right)=: K_{D}(s) .
$$

The heat kernel $K\left(x, x^{\prime} ; s\right)$ satisfies a modified heat flow equation with the following boundary conditions:

$$
\frac{\partial}{\partial s} K=\not \not^{2} K, \quad \lim _{s \rightarrow 0^{+}} K\left(x, x^{\prime} ; s\right)=\frac{\delta\left(x-x^{\prime}\right)}{\sqrt{g}} .
$$

The proper-time representation is not only useful to evaluate the functional trace of the heat kernel on curved spacetimes, but also allows to regularize this fermionic fluctuation contribution in a scale-dependent and spinbase-invariant [40] fashion: Contributions from the infrared (IR) modes of the fermionic spectrum contribute predominantly to the large-s part of the proper-time integral. Hence, these modes can be IR regularized by insertion of a regulator function $f_{k}$,

$$
f_{k}=\mathrm{e}^{-\left(k^{2} s\right)^{p}}
$$

into the proper-time integral $[41,42]$. The parameter $p>0$ specifies the renormalization scheme and $k$ corresponds to an IR regularization scale for the eigenvalues of the squared Dirac operator. For $p \rightarrow \infty$, all long-range contributions are sharply cut off at the scale $s>1 / k^{2}$. The scale $\sqrt{s}$ is a measure for the spatiotemporal range of the fluctuating modes. For finite values of $p$, the regularization scale is smeared out. In the limit $k \rightarrow 0$, the RG insertion factor becomes the identity, and the regularization is thus removed. Starting at an ultraviolet (UV) scale $k=\Lambda$ with the bare potential $\tilde{U}_{\Lambda}$, the potential in the IR at $k_{\mathrm{IR}}$ can be computed by

$\tilde{U}_{k_{\mathrm{IR}}}=\tilde{U}_{\Lambda}-\int_{k_{\mathrm{IR}}}^{\Lambda} d k \partial_{k} \tilde{U}_{k}, \quad \tilde{U}_{\Lambda}=\frac{N_{\mathrm{f}}}{2 \bar{\lambda}_{\Lambda}} \phi_{0}^{2}, \quad \bar{\lambda}_{\Lambda}:=\bar{\lambda}$.

At intermediate scales $k$, the scale-dependent effective potential $\tilde{U}_{k}$ satisfies the flow equation

$$
\partial_{k} \tilde{U}_{k}=\frac{N_{\mathrm{f}}}{2} \int_{0}^{\infty} \frac{d s}{s} \mathrm{e}^{-\phi_{0}^{2} s}\left(\partial_{k} f_{k}\right) K_{D}(s) .
$$

The advantage of performing the integral over the Schwinger proper time $s$ first is that the cutoff $\Lambda$ controls the UV divergences and thus assists one to identify and fix counterterms for the corresponding relevant and marginal operators.

\section{HEAT KERNELS}

Aiming at an analysis of the scale-dependent effective potential of Eq. (13), the information about the spacetime structure enters via the heat-kernel trace $K_{D}(s)$. As we are 
interested in the mechanism of gravitational catalysis and the influence of finite temperature, we focus on spacetimes that feature a sufficient amount of negative curvature and allow for a simple use of thermal field theory in imaginarytime formalism. Therefore, a natural choice is $S^{1} \otimes H^{d}$ with a compactified (Euclidean) time and the spatial part corresponding to a maximally symmetric hyperboloid with negative spatial curvature. The decompactified limit then corresponds to the zero-temperature case $\mathbb{R} \otimes H^{d}$ with a flat time direction.

It is important to emphasize that we do not at all consider these spacetimes as physical descriptions of the large-scale structure of the Universe. By means of our scale-dependent analysis, we focus on effective properties of quantum spacetime, say, in the trans-Planckian regime. Here, nothing specific is known about the microscopic spacetime structure. Hence, our choice of spacetime can be considered as a proxy for a possible structure of local patches of spacetime in that short-distance regime of quantum gravity. For the product manifolds considered here, the square of the Dirac operator can be decomposed as

$$
\not \not_{D}^{2}=\left(\partial_{0}\right)^{2}+\not \not_{d}^{2}, \quad D=d+1 .
$$

Correspondingly, the heat-kernel trace factorizes:

$$
K_{D}(s)=\operatorname{Tr}_{t} \mathrm{e}^{\left(\partial_{0}\right)^{2} s} \cdot \operatorname{Tr}_{x} \mathrm{e}^{\bigotimes_{d}^{2} s}=K_{t}(s) \cdot K_{d}(s) .
$$

Let us first discuss the spatial part $K_{d}(s)$ for which an analytical result exists and has been worked out for general dimensions $d$ [43]. Focusing in this work on $d=3$-dimensional space, the result is particularly simple:

$$
K_{d=3}(s)=\frac{1}{(2 \sqrt{\pi s})^{3}}\left(1+\frac{1}{2} \kappa^{2} s\right)
$$

which holds for an arbitrary curvature parameter

$$
\kappa^{2}=-\frac{R}{d(d-1)}=-\frac{R}{6}>0
$$

The temporal part depends on the circumference $\beta=\frac{1}{T}$ of the Euclidean time $S^{1}$. Using antiperiodic boundary conditions for the fermionic fields, the trace if performed in momentum space runs over Matsubara frequencies $\omega_{n}=2 \pi T(n+1 / 2)$, yielding

$$
K_{t}(s)=T \sum_{n=-\infty}^{\infty} \mathrm{e}^{-\omega_{n}^{2} s}=T \vartheta_{2}\left(0, \mathrm{e}^{-(2 \pi T)^{2} s}\right)
$$

Here we encounter the Jacobi theta function $\vartheta_{2}(z, q)$. For our purposes, a Poisson resummation connecting $\vartheta_{2}$ to $\vartheta_{3}$ is useful for later numerical evaluation. It also gives direct access to analytic studies of the low-temperature limit implying the decompactification $S^{1} \rightarrow \mathbb{R}$ of the Euclidean time direction,

$$
\begin{aligned}
K_{t}(s) & =T \vartheta_{2}\left(0, \mathrm{e}^{-(2 \pi T)^{2} s}\right) \\
& =\frac{\sqrt{\pi}}{\sqrt{(2 \pi)^{2} s}} \vartheta_{3}\left(\frac{\pi}{2}, \mathrm{e}^{-\frac{\pi^{2}}{(2 \pi T)^{2} s}}\right) \\
& =\frac{1}{\sqrt{4 \pi s}}\left[1-2 \mathrm{e}^{-\frac{1}{4 T^{2} s}}+\mathcal{O}\left(\left(\mathrm{e}^{-\left(4 T^{2} s\right)^{-1}}\right)^{2}\right)\right] .
\end{aligned}
$$

Here, we obtain the standard zero-temperature results $K_{t}(s)=1 / \sqrt{4 \pi s}$ for a fully decompactified temporal direction.

\section{CURVATURE BOUNDS}

We are now in a position to derive bounds on the curvature parameter that characterize the parameter space free of gravitational catalysis. For this, we follow the reasoning of [33] and monitor the possible occurrence of nontrivial minima of the effective potential for the chiral order parameter $\phi_{0}$. In addition to the divergencies associated with matter operators to be renormalized (see next subsection), the effective potential $\tilde{U}\left(\phi_{0}\right)$ displayed, e.g., in Eq. (8), also contains a divergent zero-point energy, which we subtract by defining

$$
U\left(\phi_{0}\right)=\tilde{U}\left(\phi_{0}\right)-\tilde{U}(0)
$$

such that $U(0)=0$ is fixed at the origin in field space [44]. A possible mixing of the subtraction terms with the cosmological-constant term is not considered in this work; we assume the-possibly scale-dependent-behavior of the cosmological constant to be provided by a given quantum-gravity scenario (including matter backreactions).

To be more precise, our considerations can make direct contact with quantum-gravity scenarios, provided that such a scenario allows for an effective description of spacetime in terms of (pseudo-)Riemannian manifolds with a potentially scale-dependent notion of effective curvature arising by suitably averaging over local patches of spacetime. In the course of the following considerations, we assume all gravity-related parameters to be provided by some quantum-gravity scenario; in addition to an effective curvature, this includes potential further gravity-matter couplings, as well as the corresponding scale dependence of these quantities. In our approach, we will ignore a possible direct contribution of gravity fluctuations to the matter couplings, e.g., to $\bar{\lambda}$; however, such contributions have been found to be less relevant for the status of chiral symmetry of the matter sector $[1,3]$.

\section{A. Curvature bounds at zero temperature}

Let us first work out the renormalization of the effective potential, identifying all free parameters by accordingly 
fixing the required renormalization counterterms. Using the preceding results for the heat-kernel traces, the zerotemperature effective potential of Eq. (8) upon IR regularization (11) and zero-point subtraction (19) reads

$$
\begin{aligned}
U_{k}= & \frac{N_{\mathrm{f}}}{2 \bar{\lambda}} \phi_{0}^{2} \\
& +\frac{N_{\mathrm{f}}}{2(4 \pi)^{2}} \int_{0}^{\infty} \frac{d s}{s^{3}} f_{k}\left(\mathrm{e}^{-\phi_{0}^{2} s}-1\right)\left(1+\frac{1}{2} \kappa^{2} s\right) .
\end{aligned}
$$

A power-counting analysis reveals the occurrence of a quadratic divergence for the $\phi_{0}^{2}$ operator and two logarithmic divergences for the $\phi_{0}^{4}$ and $\phi_{0}^{2} R$ operators, respectively.

As a sufficient criterion for the occurrence of chiral symmetry breaking, we specifically monitor the sign of the $\phi_{0}^{2}$ term in the Taylor expansion of the effective potential. If this sign turns negative, the $\phi_{0}^{4}$ operator cannot inhibit chiral symmetry breaking. For the curvature bound derived below, the $\phi_{0}^{4}$ operator is thus not relevant; from here on, we assume it to be properly renormalized such that the coupling has some finite value at the scale $k$ at which we consider the theory. We add that the sign criterion of the $\phi_{0}^{2}$ is not a necessary criterion for chiral symmetry breaking, as first-order-type transitions to a broken phase could go along with a positive $\phi_{0}^{2}$ term. We ignore this option in the following; if it was realized, our curvature bound would even get stronger.

The remaining divergences can conveniently be identified by using the flow equation (13), inserting the regulator (11) and expanding in $\phi_{0}$. To leading order, we obtain

$$
\partial_{k} U_{k}=\frac{k N_{\mathrm{f}} \phi_{0}^{2}}{2(4 \pi)^{2}}\left[2 \Gamma\left(1-\frac{1}{p}\right)+\frac{\kappa^{2}}{k^{2}}\right]+\mathcal{O}\left(\phi_{0}^{4}\right) .
$$

Here, we observe a divergence for the case of a regularization parameter $p=1$.

This is expected, as this value would correspond to a mass-type Callan-Symanzik regularization scheme which is known to be insufficient for an adequate suppression of UV modes in four dimensions. In order not to be affected by this artificial divergence from the regulator, we suggest to use schemes with $p \geq 2$.

Next, we integrate the flow from an IR scale $k_{\mathrm{IR}}$ to a UV scale $\Lambda$, using for the UV boundary condition not only the flat space expression as in Eq. (12), but also including a possible scalar-curvature counterterm,

$$
U_{\Lambda}\left(\phi_{0}\right)=\frac{N_{\mathrm{f}}}{2 \bar{\lambda}_{\Lambda}} \phi_{0}^{2}+N_{\mathrm{f}} \xi_{\Lambda} \phi_{0}^{2} R
$$

with a UV coupling $\xi_{\Lambda}$. The resulting effective potential at $k=k_{\mathrm{IR}}$ then reads up to order $\phi_{0}^{2}$ and ignoring terms of order $\mathcal{O}(1 / \Lambda)$ :

$$
\begin{aligned}
U_{k_{\mathrm{IR}}}= & -\frac{N_{\mathrm{f}} \phi_{0}^{2}}{2}\left(\frac{1}{\bar{\lambda}_{\mathrm{cr}}}-\frac{1}{\bar{\lambda}_{\Lambda}}-\frac{k_{\mathrm{IR}}^{2}}{16 \pi^{2}} \Gamma\left(1-\frac{1}{p}\right)\right) \\
& -6 N_{\mathrm{f}} \xi_{k_{\mathrm{IR}}} \phi_{0}^{2} \kappa^{2}+\mathcal{O}\left(\phi_{0}^{4}\right) .
\end{aligned}
$$

Here, we have introduced the (scheme-dependent) critical coupling of the chiral channel

$$
\bar{\lambda}_{\mathrm{cr}}=\frac{16 \pi^{2}}{\Lambda^{2} \Gamma\left(1-\frac{1}{p}\right)}
$$

and defined the finite scalar-curvature coupling at the scale $k_{\mathrm{IR}}$ as

$$
\xi_{k_{\mathrm{IR}}}=\xi_{\Lambda}+\frac{1}{12(4 \pi)^{2}} \log \left(\frac{\Lambda}{k_{\mathrm{IR}}}\right) .
$$

In this work, we consider $\xi_{k_{\mathrm{R}}}$ to be a free parameter to be determined by the underlying quantum-gravity theory. Equation (23) can be interpreted as follows: The first line contains the information about the symmetry status in flat spacetime. In a subcritical regime, e.g., $\bar{\lambda}_{\Lambda}<\bar{\lambda}_{\text {cr }}$, the masslike term remains positive for zero curvature, indicating that the origin, $\phi_{0}=0$, is a local minimum of the potential (in fact, it is also a global one); hence the system is in the disordered phase and the fermion mass remains zero. In the supercritical regime however, e.g., $\bar{\lambda}_{\Lambda}>\bar{\lambda}_{\text {cr }}$, the masslike term in the first line can become negative for decreasing $k_{\mathrm{IR}}$ resulting in a nontrivial minimum $\phi_{0}^{2}>0$ in the long-range limit. This implies chiral symmetry breaking and fermion mass generation in flat spacetime. Now, the second line of Eq. (23) contains the curvature contributions resulting from the hyperbolically curved space. Assuming $\xi_{k_{\mathrm{IR}}}$ to be positive, the prefactor of this second term is negative and can therefore cause chiral symmetry breaking depending on the magnitude of the terms in the first line. Of course, we assume the fermionic self-interactions to be subcritical; otherwise the system would be in an NJL-like phase which does not conform with the low-mass scale of the Standard-Model fermions. While finite values of $\bar{\lambda}_{\Lambda}$ are expected to be generated by gauge and Yukawa interactions, we use the following simple estimate for the first line of Eq. (23):

$$
\begin{gathered}
-\frac{\phi_{0}^{2}}{2}\left(\frac{1}{\bar{\lambda}_{\mathrm{cr}}}-\frac{1}{\bar{\lambda}_{\Lambda}}-\frac{k_{\mathrm{IR}}^{2}}{16 \pi^{2}} \Gamma\left(1-\frac{1}{p}\right)\right) \\
\geq \phi_{0}^{2} \frac{k_{\mathrm{IR}}^{2}}{32 \pi^{2}} \Gamma\left(1-\frac{1}{p}\right) .
\end{gathered}
$$

Comparing this to the curvature-dependent contribution $\sim \xi_{k_{\mathrm{IR}}}$, we conclude that gravitational catalysis does not occur, if the ratio of the curvature of local patches of spacetime to the energy scale satisfies 


$$
\frac{\kappa^{2}}{k_{\mathrm{IR}}^{2}} \leq \frac{\Gamma\left(1-\frac{1}{p}\right)}{192 \pi^{2} \xi_{k_{\mathrm{IR}}}}
$$

Any finite value of the fermionic self-interaction $\bar{\lambda}_{\Lambda}$ at the high scale would even strengthen the bound. We observe an apparent explicit scheme dependence of our bound through the regularization parameter $p$. For the region $2 \leq p<\infty$, this dependence is rather mild, since $1<\Gamma\left(1-\frac{1}{p}\right) \leq \sqrt{\pi}$. However, it should be noted that also the left-hand side carries an implicit scheme dependence, since the dimensionless ratio of curvature-which we consider as an effective curvature of spacetime patches-and the IR scale $k_{\mathrm{IR}}$ depend on the details of the spacetime averaging procedure. As the latter, if done explicitly, would go hand in hand with the average over the fermionic fluctuations on various length scales, we expect the existence of such a bound as in Eq. (27) to have a universal meaning. We take the residual $p$ dependence of (27) as a measure for our ignorance of the details of the averaging process. It is instructive to compare this result for the $\mathbb{R} \otimes H^{3}$ background with the corresponding bound for the maximally symmetric case $H^{4}$. Here, the heat-kernel trace is a nonpolynomial function of the curvature leading to an integral representation of the curvature bound [33]. For the purpose of the present discussion, we use the simple analytic approximation also given in [33]:

$$
H^{4}: \frac{\kappa^{3}}{k_{\mathrm{IR}}^{3}}+\frac{4}{3} \frac{\pi^{\frac{5}{2}}}{\Gamma\left(1+\frac{1}{2 p}\right)} \xi_{k_{\mathrm{IR}}} \frac{\kappa^{2}}{k_{\mathrm{IR}}^{2}} \leq \frac{\sqrt{\pi}}{2} \frac{\Gamma\left(1-\frac{1}{p}\right)}{\Gamma\left(1+\frac{1}{2 p}\right)} .
$$

Apart from numerical factors, the main difference arises from the first term $\sim \kappa^{3}$ in the $H^{4}$ case which is present independently of the marginal scalar-curvature coupling $\sim \xi$. Though the curvature bound itself does depend on the precise value of $\xi_{k_{\mathrm{IR}}}$ also in $H^{4}$, there is a meaningful bound for any value of, say, $\xi_{k_{\mathrm{IR}}} \sim \mathcal{O}(1)$ with $\xi_{k_{\mathrm{IR}}}=0$ being a legitimate choice. This is not the case for our present result (27) for the bound which depends strongly on $\xi_{k_{\mathrm{IR}}}$, yielding no meaningful result for $\xi_{k_{\mathrm{IR}}}=0$. The reason for this strong dependence lies in the fact that the heat-kernel trace on $H^{3}$ has the particularly simple polynomial form given in Eq. (16), the contribution of which to the effective potential can be fully absorbed in the renormalization of the marginal scalar-curvature coupling $\xi$.

We draw the following conclusions from this observation: First, this strong qualitative and quantitative difference between the curvature bounds of two example spacetimes with negative curvature demonstrates that the details of the average spacetime structure in the (trans-)Planckian regime of quantum gravity can take a strong influence on the presence or absence of gravitational catalysis. If a bound derived for one case is satisfied, it may still be violated in another case. Since we have little access to general knowledge about the average spacetime structure in this short-distance regime where spacetime itself is expected to be strongly fluctuating, the exclusion of gravitational catalysis in order to reach compatibility with the existence of light fermions can thus be decisive criterion for the viability of a quantum-gravity scenario.

Second, in addition to information about the averaged spacetime structure of local spacetime patches, a quantumgravity (plus matter) scenario has to provide also a prediction of the scalar-curvature coupling $\xi$ in order to test for gravitational catalysis. Since the scalar field in the present analysis arises from fermion interactions which may arise predominantly from classically scale-invariant gauge interactions, the use of a conformally coupled scalar field is a reasonable first guess.

\section{B. Curvature bounds at finite temperature}

As in the zero-temperature case, we now derive curvature bounds from the effective potential for the chiral order parameter. For this, we write the regularized effective potential as

$$
U_{k}^{T}=U_{k}+\Delta_{T} U_{k}
$$

where $U_{k}$ denotes the zero-temperature part [cf. Eq. (20)] and $\Delta_{T} U_{k}$ is the thermal correction satisfying $\Delta_{T=0} U_{k}=0$. Based on the heat-kernel traces, this thermal part can be written as

$$
\begin{aligned}
\Delta_{T} U_{k}= & \frac{N_{\mathrm{f}}}{2(4 \pi)^{2}} \int_{0}^{\infty} \frac{d s}{s^{3}} f_{k}\left(\mathrm{e}^{-\phi_{0}^{2} s}-1\right)\left(1+\frac{1}{2} \kappa^{2} s\right) \\
& \times\left[\vartheta_{3}\left(\frac{\pi}{2}, \mathrm{e}^{-\frac{\pi^{2}}{(2 \pi T)^{2} s}}\right)-1\right] .
\end{aligned}
$$

Since the presence of finite temperature does not modify the UV behavior of the theory, this expression is already finite. No further counterterms are required, and we consider all physical parameters to be fixed by the $T=0$ renormalization conditions. After the substitution $\tilde{s}=k_{\mathrm{IR}}^{2} s$, the thermal correction to the effective potential up to quadratic order in $\phi_{0}$ reads

$\Delta_{T} U_{k}=\frac{N_{\mathrm{f}}}{32 \pi^{2}}\left[A^{p}(\zeta) \cdot k_{\mathrm{IR}}^{2}+C^{p}(\zeta) \cdot \kappa^{2}\right] \phi_{0}^{2}, \quad \zeta=\frac{T}{k_{\mathrm{IR}}}$,

with the temperature-dependent coefficients functions

$$
\begin{aligned}
& A^{p}(\zeta)=-\frac{1}{2} \int_{0}^{\infty} \frac{d \tilde{s}}{\tilde{s}^{2}} \mathrm{e}^{-\tilde{s}^{p}}\left[\vartheta_{3}\left(\frac{\pi}{2}, \mathrm{e}^{-\frac{1}{4 \zeta^{2} \tilde{s}}}\right)-1\right], \\
& C^{p}(\zeta)=-\frac{1}{4} \int_{0}^{\infty} \frac{d \tilde{s}}{\tilde{s}} \mathrm{e}^{-\tilde{s}^{p}}\left[\vartheta_{3}\left(\frac{\pi}{2}, \mathrm{e}^{-\frac{1}{4 \zeta^{2} \tilde{s}}}\right)-1\right]
\end{aligned}
$$


that depend on the regularization scheme parameter $p$ and the rescaled temperature $\zeta=T / k_{\mathrm{IR}}$. Both functions vanish in the zero-temperature limit, $A^{p},\left.C^{p}\right|_{\zeta \rightarrow 0}=0$ for any legitimate scheme parameter $p$. The quadratic part of the effective potential at finite temperature can be expressed through these coefficients functions

$$
\begin{aligned}
U_{k_{\mathrm{IR}}}^{T}= & -\frac{N_{\mathrm{f}} \phi_{0}^{2}}{2}\left[\frac{1}{\bar{\lambda}_{\mathrm{cr}}}-\frac{1}{\bar{\lambda}_{\Lambda}}-\frac{k_{\mathrm{IR}}^{2}}{16 \pi^{2}}\left(\Gamma\left(1-\frac{1}{p}\right)+A^{p}(\zeta)\right)\right] \\
& -N_{\mathrm{f}} \kappa^{2}\left(6 \xi_{k_{\mathrm{IR}}}-\frac{1}{32 \pi^{2}} C^{p}(\zeta)\right) \phi_{0}^{2}+\mathcal{O}\left(\phi_{0}^{4}\right),
\end{aligned}
$$

leading to the temperature-dependent curvature bound

$$
\frac{\kappa^{2}}{k_{\mathrm{IR}}^{2}} \leq B^{p}(\zeta):=\frac{\Gamma\left(1-\frac{1}{p}\right)+A^{p}(\zeta)}{192 \pi^{2} \xi_{k_{\mathrm{IR}}}-C^{p}(\zeta)} .
$$

This bound represents a central result of our work. The integrals for the coefficients $A^{p}(\zeta)$ and $C^{p}(\zeta)$ can be evaluated numerically for arbitrary $p$ rather straightforwardly. For analytic estimates, we expand the thermal part of the heat kernel, excluding the zero-temperature contribution, in a Taylor expansion for the second argument of the Jacobi theta function

$$
\left[\vartheta_{3}\left(\frac{\pi}{2}, \mathrm{e}^{-\frac{\pi^{2}}{(2 \pi T)^{2} s}}\right)-1\right]=2 \sum_{n=1}^{\infty}(-1)^{n} \mathrm{e}^{-\frac{n^{2}}{4 \xi^{2} s}}
$$

We observe that the contributions decrease exponentially for each additional order suggesting that expansions truncated at a certain order $N$ can still represent a quantitatively accurate approximation up to a certain temperature. Expanding the thermal coefficients from Eq. (32) accordingly, we can express the result to all orders in the expansion by the two functions $a^{p}(z)$ and $c^{p}(z)$, respectively,

$$
\begin{aligned}
A^{p}(\zeta) & =-\sum_{n=1}^{\infty}(-1)^{n} \int_{0}^{\infty} \frac{d \tilde{s}}{\tilde{s}^{2}} \mathrm{e}^{-\tilde{s}^{p}} \mathrm{e}^{-\frac{n^{2}}{4 \zeta^{2} \tilde{s}}} \\
& =: \sum_{n=1}^{\infty}(-1)^{n} a^{p}(\zeta / n), \\
C^{p}(\zeta) & =-\frac{1}{2} \sum_{n=1}^{\infty}(-1)^{n} \int_{0}^{\infty} \frac{d \tilde{s}}{\tilde{s}} \mathrm{e}^{-\tilde{s}^{p}} \mathrm{e}^{-\frac{n^{2}}{4 \zeta^{2} \tilde{s}}} \\
& =: \sum_{n=1}^{\infty}(-1)^{n} c^{p}(\zeta / n) .
\end{aligned}
$$

These functions can be computed analytically for the scheme parameters $p=1$ and $p=\infty$ and yield

$$
\begin{aligned}
& a^{p=1}(z)=-8 z K_{1}\left(\frac{1}{z}\right), \\
& a^{p=\infty}(z)=-8 z^{2} \mathrm{e}^{-\frac{1}{4 z^{2}}}, \\
& c^{p=1}(z)=-2 K_{0}\left(\frac{1}{z}\right), \\
& c^{p=\infty}(z)=\operatorname{Ei}\left(-\frac{1}{4 z^{2}}\right),
\end{aligned}
$$

with $K_{n}(z)$ being the modified Bessel functions of the second kind and $\operatorname{Ei}(z)$ the exponential integral. Whereas the choice $p=1$, corresponding to the Callan-Symanzik regulator, is insufficient for regularizing the quantum fluctuations as discussed above, there is no problem using it for the thermal part. While setting $p=2$ for the quantum and $p=1$ for the thermal fluctuations does not correspond to a fully consistent regularization scheme, the comparison between $p=\infty$ and the " $p=1,2$ " scheme can be used for analytical estimates of the scheme dependence. A full numerical comparison between the extreme choices $p=2$ and $p=\infty$ is shown in Fig. 1. Here, the bound $B^{p}(\zeta)$ of Eq. (35) is shown as a function of rescaled temperature for the two schemes. While there is a quantitative difference for low temperatures which reflects the scheme dependences found in Eq. (27) for $T=0$, this difference significantly weakens for increasing temperature. This enhances the predictivity of our quantitative estimates for the finitetemperature case.

A fully analytical estimate is obtained by truncating the series in Eqs. (37) and (38) at a finite order in $N$ using, say, the $p \rightarrow \infty$ scheme. In Fig. 2, we compare increasing orders for $N=1,3,5$ with the corresponding full numerical result. We observe that already low-order estimates reflect the full behavior qualitatively rather well. For increasing order, also the quantitative precision increases. For instance, for $N=15$ no difference between the analytical estimate and the numerical result would be

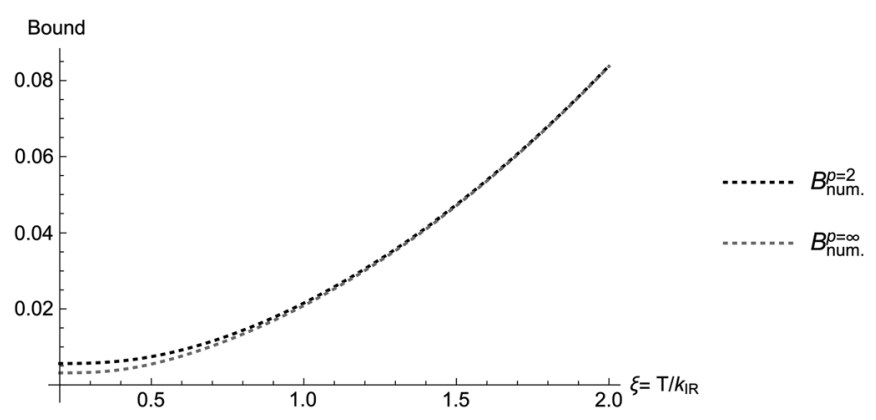

FIG. 1. Numerical result for the curvature bound $B^{p}(\zeta)$ of Eq. (35) as a function of the rescaled temperature $\zeta=T / k_{\mathrm{IR}}$ for regularization-scheme parameters $p=2$ and $p=\infty$, respectively. The comparatively mild scheme dependence at zero temperature even weakens for increasing temperature. 


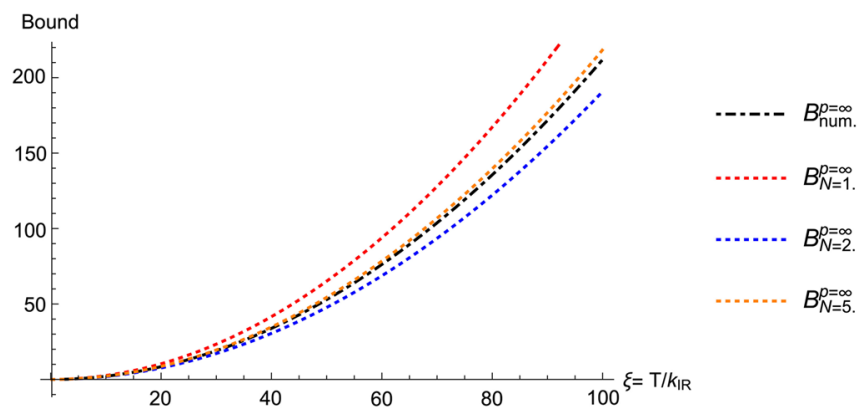

FIG. 2. Numerical result for the curvature bound $B^{p \rightarrow \infty}(\zeta)$ of Eq. (35) as a function of the rescaled temperature $\zeta=T / k_{\mathrm{IR}}$ in comparison with the analytical estimates of Eqs. (37) and (38) for increasing truncations $N$. Even for large values of $\zeta$, the analytical estimates approach the full result rather rapidly. For large temperatures, the curvature bound increases $\sim \zeta^{2}$.

visible in Fig. 2 in the shown regime of rescaled temperatures as large as $\zeta=100$. The large- $\zeta$ behavior of the bound fits well to quadratic increase. A numerical fit yields $B^{p}(\zeta) \simeq 0.02 \zeta^{2}$ for the leading high-temperature behavior. This matches also with the qualitative behavior of the largetemperature expansion of the heat kernel.

For the application of our curvature bound to a quantumgravity scenario below, we simply use the analytical estimate $B^{p \rightarrow \infty}(\zeta)$ for $N=15$, as it is sufficiently accurate for all values of rescaled temperature $\zeta$ of interest.

\section{ASYMPTOTICALLY SAFE GRAVITY: FROM CURVATURE BOUND TO MATTER BOUND}

The preceding results can be applied to generic quantumgravity scenarios as soon as they feature an effective metric-based description below a certain high-energy scale. For this, we assume that such a scenario provides information about the effective spacetime structure at shortdistance scales, e.g., in the form of a possibly scaledependent effective metric, $\left\langle g_{\mu \nu}\right\rangle_{k}$. In addition, we assume that the quantum-gravity scenario also accommodates a model of the cosmological evolution going along with a scale-dependent evolution of the temperature. In fact, the asymptotic-safety scenario for quantum gravity [45-48] has witnessed rapid progress over the past two decades, as, e.g., reviewed in [49-58], and is thus able to provide us with required estimates also including matter degrees of freedom $[5,9,32,59-68]$. The scenario therefore serves as an example in the following. Let us briefly summarize the corresponding line of argument developed in [33], generalizing it to the presence of finite temperature during a cosmological evolution. For simplicity, we work in the socalled Einstein-Hilbert truncation, assuming that higherorder curvature operators-though relevant for a more accurate picture of the UV behavior [60,69-81]—do not take a strong influence on the RG trajectory at the effective scales considered here. Incidentally, this approximation could straightforwardly be improved, e.g., by considering trajectories as in [82]. The effective scale-dependent metric obeys the quantum equation of motion whichon the Einstein-Hilbert level—corresponds to Einstein's equations,

$$
R_{\mu \nu}\left(\langle g\rangle_{k}\right)-\frac{1}{2} R\left(\langle g\rangle_{k}\right)\left\langle g_{\mu \nu}\right\rangle_{k}+\bar{\Lambda}_{k}\left\langle g_{\mu \nu}\right\rangle_{k}=0 .
$$

Within the asymptotic-safety scenario, the dimensionless version of the scale-dependent cosmological parameter $\bar{\Lambda}_{k}$ is governed by the Reuter fixed point, i.e., a non-Gaußian UV fixed point $\lambda_{*}$, in the trans-Planckian region of the RG flow. Even though typical RG trajectories appear to spiral around the fixed point toward the UV, i.e., quantitatively relevant values potentially oscillate about $\lambda_{*}$ during the course of the RG evolution, we use this fixed-point value as an estimate for the effective curvature of local spacetime patches averaged over a length scale $\sim 1 / k_{\mathrm{IR}}$. Since the background $S^{1} \otimes \mathbb{W}^{3}$ chosen for our finite-temperature analysis is not a solution to the Einstein equation (43), i.e., it is not of Friedmann-Lemaitre type, we cannot unambiguously link our background-curvature parameter $\kappa$ to the fixed-point value $\lambda_{*}$ of the asymptotic-safety scenario. In the following, we use the trace of the Einstein equation, which yields in the fixed-point regime:

$$
\frac{R}{k_{\mathrm{IR}}^{2}}=4 \lambda_{*}
$$

Alternatively, we could use solely the spatial components of the Einstein equation for which $\mathbb{M}^{3}$ is a solution; in this case, a factor of 6 would replace the factor of 4 on the righthand side of Eq. (44), mildly modifying our quantitative results below. In the following, we use the trace prescription leading to Eq. (44), as it implements isotropy on the level of the equation of motion. By means of this relation, the asymptotic-safety scenario relates the curvature of local spacetime patches in the trans-Planckian regime to the fixed-point value of the cosmological parameter. In those regimes where the latter is positive our curvature bounds are irrelevant, as they are automatically fulfilled. Hence, we concentrate on the case where $\lambda_{*}<0$, for which we obtain an estimate for our curvature parameter:

$$
\frac{\kappa^{2}}{k^{2}}=\frac{2\left|\lambda_{*}\right|}{3}>0, \quad \text { for } \lambda_{*}<0
$$

A crucial observation within the asymptotic-safety scenario is that the fixed-point properties depend on the matter content $[59,61,83]$, i.e., on the nature of the fluctuating quantum degrees of freedom coupling to gravity. In the present setting, the dependence of $\lambda_{*}$ on this matter content comes in through two parameter combinations: 


$$
d_{g}=N_{\mathrm{S}}-4 N_{\mathrm{V}}+2 N_{\mathrm{f}}, \quad d_{\lambda}=N_{\mathrm{S}}+2 N_{\mathrm{V}}-4 N_{\mathrm{f}},
$$

where $N_{\mathrm{S}}$ counts the number of scalar degrees of freedom, $N_{\mathrm{V}}$ denotes vector degrees of freedom, and $N_{\mathrm{f}}$ is the flavor number as before. (Here, we quote results for the so-called type IIa regulator [52] which accounts for the appropriate endomorphisms of the Laplacians for particles with spin [61].) The precise dependence of $\lambda_{*}$ on these matter parameters is not yet fully determined. Current results show some dependence on the details of the nonperturbative approximation; see, e.g., $[3,52,61,84]$. A quantitative comparison concerning gravitational catalysis in the zerotemperature limit can be found in [33]. Roughly speaking, $\lambda_{*}$ in simple approximations is proportional to $d_{\lambda}$, such that a dominant number of fermion flavors $N_{\mathrm{f}}$ moves the system toward the region where gravitational catalysis could become relevant. For the following quantitative discussion, we use the fixed-point results of [84] and their dependence on $d_{g}$ and $d_{\lambda}$ as an example. We focus on the regularization scheme $p \rightarrow \infty$ and — unless stated otherwise-assume the scalar-curvature coupling at its conformally coupled point $\xi_{k_{\mathrm{IR}}}=1 / 6$ which is known to be a fixed point of the universal part of the perturbative RG [68,85-87]; the dependence of our quantitative results on $\xi_{k_{\mathrm{IR}}}$ is also studied below. In order to complete the concrete scenario of our investigation, we need to connect the scale $k_{\mathrm{IR}}$ at which we consider the system with a value (or range of values) for the temperature $T$. In a specific cosmological model, the temperature would be connected with a relevant cosmological scale, say, a time parameter or an expansion scale. Within asymptotically safe cosmologies, such scales are assumed to be linked to some suitable power of $k$ by RG-improvement arguments [88-96]. In fact, several scalesetting procedures have been discussed in the literature [89,96-98]. For the present study, we therefore use the rescaled temperature $\zeta=T / k_{\mathrm{IR}}$ as a parameter; the value (or range of relevant values) will be fixed by a specific choice of the cosmological model. Simple RG-improvement arguments suggest to consider $\zeta \sim \mathcal{O}(1)$. Since our zero-temperature bound on $\mathbb{R} \otimes H^{3}$ is quantitatively stronger than the corresponding one on $H^{4}$ for $\xi_{k_{\mathbb{R}}}=0$ as used in [33], we expect a correspondingly larger extent of the regime where gravitational catalysis could be active. Given our result that the curvature bound (35) weakens for increasing temperature, the region which is not affected by gravitational catalysis should increase with $\zeta$. In fact, this is visible in Fig. 3: Here the orange region in the upper part of the plot indicates the region where $\lambda_{*}$ is positive in the asymptotic-safety scenario; hence this region is not affected by gravitational catalysis.

At finite rescaled temperature $\zeta=T / k_{\mathrm{IR}}$, the solid lines separate the regions in this space of asymptotically safe theories with matter which are free of gravitational catalysis (regions above and left of lines) from those where our curvature bound is violated and gravitational catalysis

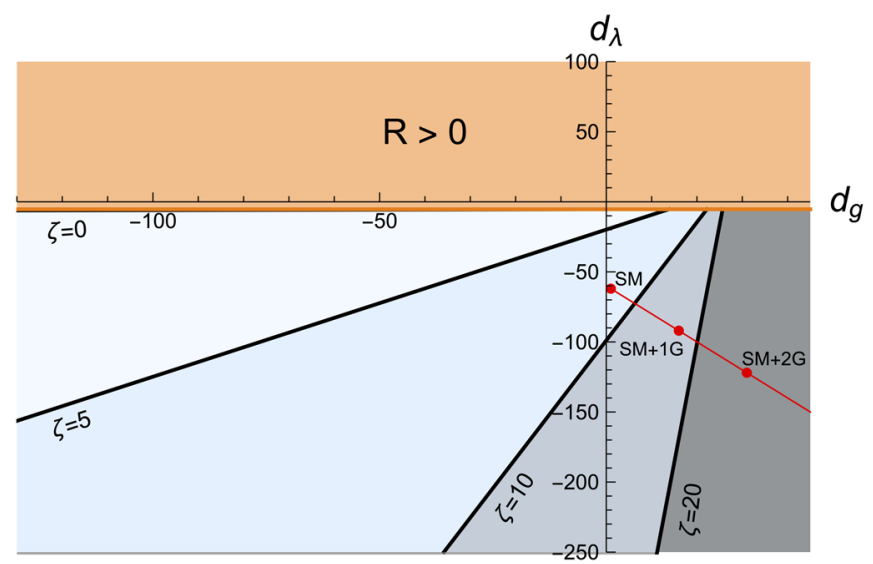

FIG. 3. Space of asymptotically safe quantum-gravity theories with matter parametrized by $d_{g}$ and $d_{\lambda}$ according to Eq. (46). The orange area corresponds to regions with positive curvature. Each of the four solid lines distinguish regions free from gravitational catalysis (region above and left of each line) from regions that violate our curvature bound and could feature chiral symmetry breaking through gravitational catalysis (darker shaded region below and right of each line)_for the rescaled temperatures $\zeta=0$ (barely visible in the upper left sector), 5, 10, and 20. The red dot marks the SM matter content with the red line indicating the Standard Model with additional fermionic generations.

could trigger fermion mass generation (darker shaded regions below and right of lines). In fact, the curvature bound for $\zeta=0$ is rather close to the $R>0$ curve with only a slim unaffected region extending along the negative $d_{g}$ axis (hardly visible on the scale of this Fig. 3). This agrees with the comparatively strong curvature bound on $\mathbb{R} \otimes H^{3}$ for $\xi_{k_{\mathrm{IR}}}=1 / 6$ and should be taken as an indication that gravitational catalysis might be more relevant than previously anticipated for the $\mathrm{H}^{4}$ background. In other words, the details of the spacetime structure of local spacetime patches do matter beyond the simple statement of positive or negative average curvature and thus need to be addressed by the quantum-gravity scenario under scrutiny.

For increasing rescaled temperature $\zeta$ the region satisfying the curvature bound increases; for $\zeta>\mathcal{O}(10)$, the boundary line approaches a vertical line that ultimately matches with a region where the computation of [84] does no longer find a viable UV fixed point.

It is interesting to observe that the Standard-Model (SM) matter content with three generations and thus $N_{\mathrm{S}}=4$, $N_{\mathrm{V}}=12$ and $N_{\mathrm{f}}=45 / 2$ (excluding right-handed neutrino components) (red dot in Fig. 3) lies in the region violating the bound for small $\zeta$ but satisfying the bound for $\zeta>8.3$ for the current assumptions. This illustrates directly that a given quantum-gravity scenario does not automatically allow for an arbitrary matter content. Depending on the details of the local spacetime curvature, gravitational catalysis could be relevant and needs to be carefully scrutinized in this regime. 
At the same time, our current study also reveals how gravitational catalysis endangering the existence of light fermions could be tamed in the course of the cosmological evolution: Even a critical spacetime curvature violating the zero-temperature bound may not give rise to gravitational catalysis and fermion mass generation provided the temperature remains sufficiently high compared to the averaging scale $k_{\mathrm{IR}}$. From an RG perspective, this can be understood in terms of the thermal masses of the fermions, which effectively suppress the fermionic fluctuations. This inhibits the symmetry-breaking channels to become RG relevant as predicted by zero-temperature catalysis. A similar mechanism has been investigated in scenarios of Higgs inflation in order not to be affected by further minima in the Higgs potential [99].

This argument can also be inverted: In order to evade gravitational catalysis for a given matter content in asymptotically safe gravity, the cosmological evolution in the early Universe has to go along with a sufficiently high (rescaled) temperature. In this way, gravitational catalysis can put bounds on the cosmological model.

As we parametrize such models using the rescaled temperature, a given value of $\zeta$-which should be understood as a lowest value in a given model in the early Universe - can accommodate a certain matter content. In order to illustrate this dependence, we concentrate on Standard-Model-like theories possibly with extra generations of fermions. In Fig. 3, these theories move along the red line toward the region increasingly endangered by gravitational catalysis with the cases of additional complete generations (" $+1 G$ " and " $+2 G$ ") marked by red dots.

By virtue of the fixed-point structure, an arbitrarily large number of fermions is not supported. This is visible in Fig. 4, where the allowed number of fermions $N_{\mathrm{f}}$ compatible with our bound is plotted as a function of $\zeta$.

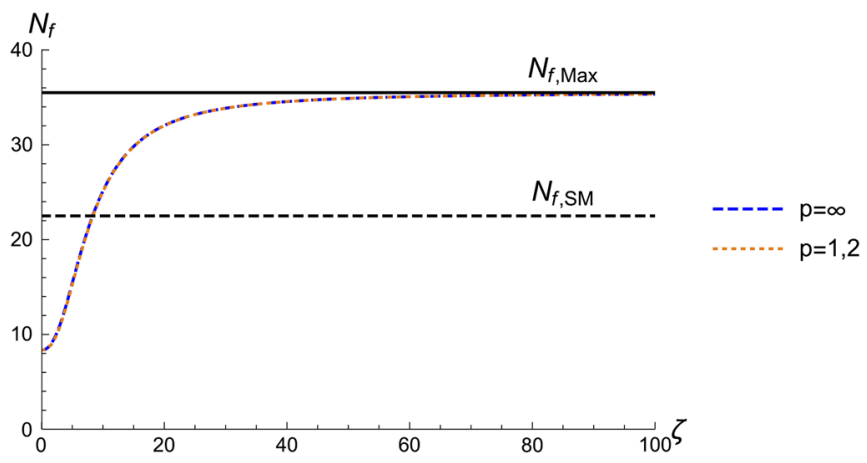

FIG. 4. Number of fermion species for a Standard-Model-like particle content $\left(N_{\mathrm{S}}=4, N_{\mathrm{V}}=12\right)$ compatible with the curvature bound from gravitational catalysis as a function of the rescaled temperature $\zeta$ for different regularization schemes $p$ and the scalar-curvature coupling $\xi_{k_{\mathrm{IR}}}=1 / 6$. The solid black line represents the upper bound $N_{f, \max }=35.5$ which is approached in the limit $\zeta \rightarrow \infty$; the dashed line marks the number of fermions in the Standard Model $N_{f, \mathrm{SM}}=22.5$.

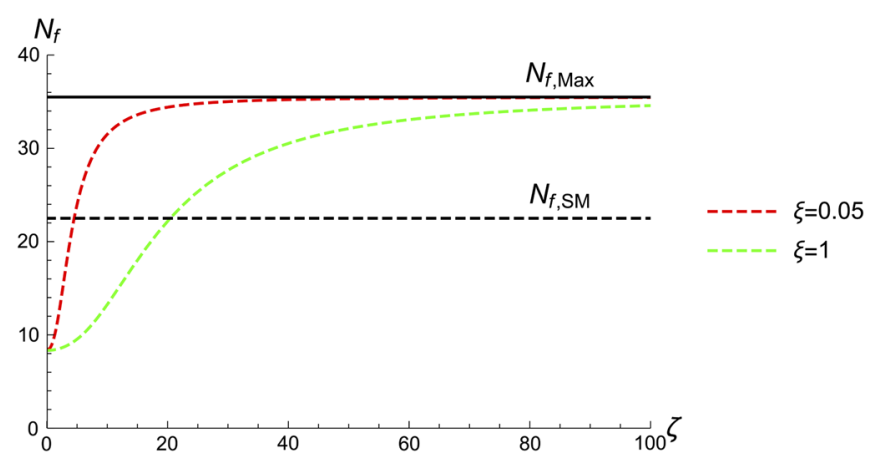

FIG. 5. Number of fermion species for a Standard-Model-like particle content $\left(N_{\mathrm{S}}=4, N_{\mathrm{V}}=12\right)$ compatible with the curvature bound from gravitational catalysis as a function of the rescaled temperature $\zeta$ for different scalar-curvature coupling parameters $\xi_{k_{\mathrm{IR}}}$ using the regularization scheme $p=\infty$. The horizontal lines are as in Fig. 4.

The observed threshold set by the Standard-Model fermion content is marked by a horizontal dashed line; it is surpassed for $\zeta>8.3$. Even at asymptotic temperatures, a maximum fermion number of $N_{\mathrm{fmax}}=35.5$ is approached. In this figure, we also illustrate the scheme dependence of our finite-temperature results by showing the extremal parameter choices $p \rightarrow \infty$ and the mixed approximate scheme $p=1,2$. On the scale of this figure, hardly any variation is recognizable, which illustrates that the scheme dependencies are under control here.

By contrast, there is a stronger dependence on the scalarcurvature coupling $\xi_{k_{\mathrm{IR}}}$. Nevertheless, while the zerotemperature bound is inversely proportional to and thus rather strongly varying with $\xi_{k_{\mathrm{IR}}}$, the finite-temperature results are somewhat less sensitive. This is visible in Fig. 5, where the number of fermions $N_{\mathrm{f}}$ that can be accommodated is shown for $\xi_{k_{\mathrm{IR}}}=0.05$ and $\xi_{k_{\mathrm{IR}}}=1$. Both curves eventually surpass the Standard-Model threshold, however for different values of the rescaled temperature.

In summary, the asymptotic-safety scenario for quantum gravity together with Standard-Model matter content can evade the curvature bound imposed by gravitational catalysis provided the temperature is sufficiently high in the course of the cosmological evolution. By contrast, theories with a more dominant fermionic matter content either require much higher temperatures to comply with the bounds or fail to support a UV-completing fixed point.

\section{CONCLUSIONS}

The present work generalizes the concept of curvature bounds from gravitational catalysis [33] to finite temperatures as well as to the case of a spatially curved space. In addition to the role played by the thermal effects, we observe that the details of the averaged curvature of local patches of spacetime matters significantly: First, gravitational catalysis is more strongly triggered for the spacetime $\mathbb{R} \otimes H^{3}$ than for 
the maximally symmetric case $H^{4}$. Second, also the dependence on the scalar-curvature coupling $\sim \xi \phi^{2} R$ is much more prominent for the former case than for the latter. Both observations have a strong influence on the curvature bound that indicates how the details of the curvature background matter for the phenomenon of gravitational catalysis.

It is important to emphasize that the curvature bound derived in this paper is an estimate for the extent of the region that is not affected by gravitational catalysis according to our assumptions. If a system (e.g., subject to a specific quantum-gravity scenario) violates the curvature bound, this does not necessarily imply that fermion mass generation kicks in as a manifestation of gravitational catalysis. Further dynamical mechanisms could still avoid the occurrence of gravitational catalysis. For instance, fluctuations of the scalar order parameter tend to weaken the symmetry-breaking channel. On the other hand, a finite initial fermionic self-interaction could enhance the tendency toward fermionic gap formation. Also, the curvature bound does not account for the possibility of further local minima which could become the global one at a first-order transition; our method is only sensitive to second-order transitions. Of course, first-order transitions could straightforwardly be detected by a global study of the effective potential. If they occur, they would strengthen our bounds.

As a first example, we have applied the curvature bound to the asymptotic-safety scenario for quantum gravity. A rather robust prediction of this scenario that relies on the existence of an interacting UV-fixed point is that the cosmological constant can have a negative sign in the shortdistance regime (with a dynamical transition to positive values for the long-range physics) depending on the matter content. In particular, a dominance of fermionic matter degrees of freedom pushes the fixed point of the cosmological term to negative values. RG-improvement arguments then suggest that the properties of the quantum spacetime in the short-distance regime can effectively be described by a scale-dependent version of Einstein's equations (or higher-derivative versions thereof). For our purposes this suggests that local patches of spacetime appear as effectively negatively curved. If so, this effective negative curvature also enhances the symmetry-breaking channels of fermionic fluctuations by (the scale-dependent version of) gravitational catalysis. If symmetry breaking was triggered in the high-energy regime of gravity, fermions would acquire a mass proportional to the scale of symmetry breaking. Gravitational catalysis would therefore inhibit the existence of light fermions in nature.

This line of argument thus connects the observational fact of light fermions with properties of quantum spacetime in the high-energy regime. By extending the RG-improvement argument to a cosmological setting, our reasoning connects the curvature bound of gravitational catalysis to a combination of matter degrees of freedom such as the fermion flavor number together with the thermal evolution of the Universe, parameterized in this work by the rescaled temperature $\zeta$.

Whereas our results for the general curvature bound have a clear quantitative meaning within the given assumptions, the application to the asymptotic-safety scenario should be considered as more qualitative because of the approximations involved and the genuine qualitative nature of $\mathrm{RG}$ improvement. Therefore, we interpret these results as an indication that the asymptotic-safety scenario for quantum gravity can indeed be compatible with the existence of light fermions; there is definitely room for evading the bounds imposed by gravitational catalysis for particle models with a matter content similar to that of the Standard Model. While our line of argument based on gravitational catalysis can put an upper bound on the number of fermionic degrees of freedom, it is interesting to see that a combination of gravity and Abelian gauge interactions of fermions can also produce a lower bound [32].

We believe that it will be highly worthwhile to check for the role of gravity, specifically gravitational catalysis, and the consistency with light fermions in other scenarios of quantum gravity as well. While our bounds can be applied in other settings, the inclusion of matter degrees of freedom is a common effort in many research directions of quantum gravity $[7,100-104]$.

\section{ACKNOWLEDGMENTS}

We thank Astrid Eichhorn, Aaron Held, Riccardo Martini, Martin Pauly, Alessia Platania and Marc Schiffer for valuable discussions. We are grateful to Frank Saueressig for making his data and Mathematica files available to us. This work has been funded by the Deutsche Forschungsgemeinschaft (DFG) under Grant No. 406116891 within the Research Training Group RTG 2522/1.
[1] A. Eichhorn and H. Gies, New J. Phys. 13, 125012 (2011).

[2] O. Zanusso, L. Zambelli, G. P. Vacca, and R. Percacci, Phys. Lett. B 689, 90 (2010).
[3] J. Meibohm and J. M. Pawlowski, Eur. Phys. J. C 76, 285 (2016).

[4] A. Eichhorn, A. Held, and J. M. Pawlowski, Phys. Rev. D 94, 104027 (2016). 
[5] A. Eichhorn and S. Lippoldt, Phys. Lett. B 767, 142 (2017).

[6] A. Eichhorn, S. Lippoldt, and M. Schiffer, Phys. Rev. D 99, 086002 (2019).

[7] S. Catterall, J. Laiho, and J. Unmuth-Yockey, Phys. Rev. D 98, 114503 (2018).

[8] G. P. De Brito, Y. Hamada, A. D. Pereira, and M. Yamada, J. High Energy Phys. 08 (2019) 142.

[9] J. Daas, W. Oosters, F. Saueressig, and J. Wang, Phys. Lett. B 809, 135775 (2020).

[10] D. Ebert, A. V. Tyukov, and V. C. Zhukovsky, Phys. Rev. D 80, 085019 (2009).

[11] I. L. Buchbinder and E. N. Kirillova, Sov. Phys. J. 32, 446 (1989).

[12] I. L. Buchbinder and E. N. Kirillova, Int. J. Mod. Phys. A 04, 143 (1989).

[13] T. Inagaki, T. Muta, and S. D. Odintsov, Mod. Phys. Lett. A 08, 2117 (1993).

[14] I. Sachs and A. Wipf, Phys. Lett. B 326, 105 (1994).

[15] E. Elizalde, S. Leseduarte, and S. D. Odintsov, Phys. Rev. D 49, 5551 (1994).

[16] E. Elizalde, S. Leseduarte, S. D. Odintsov, and Yu. I. Shilnov, Phys. Rev. D 53, 1917 (1996).

[17] S. Kanemura and H.-T. Sato, Mod. Phys. Lett. A 11, 785 (1996).

[18] T. Inagaki, Int. J. Mod. Phys. A 11, 4561 (1996).

[19] T. Inagaki and K.-I. Ishikawa, Phys. Rev. D 56, 5097 (1997).

[20] G. Miele and P. Vitale, Nucl. Phys. B494, 365 (1997).

[21] P. Vitale, Nucl. Phys. B551, 490 (1999).

[22] T. Inagaki, T. Muta, and S. D. Odintsov, Prog. Theor. Phys. Suppl. 127, 93 (1997).

[23] J. Hashida, S. Mukaigawa, T. Muta, K. Ohkura, and K. Yamamoto, Phys. Rev. D 61, 044015 (2000).

[24] E. V. Gorbar and V. P. Gusynin, Ann. Phys. (Amsterdam) 323, 2132 (2008).

[25] M. Hayashi, T. Inagaki, and H. Takata, arXiv:0812.0900.

[26] T. Inagaki and M. Hayashi, Strong coupling gauge theories in LHC era, in Proceedings, International Workshop, SCGT 09, Nagoya, Japan, December 8-11, 2009 (2011), pp. 184-190.

[27] E. V. Gorbar, Phys. Rev. D 61, 024013 (1999).

[28] E. V. Gorbar, Ukr. Phys. J. 54, 541 (2009).

[29] A. Flachi and K. Fukushima, Phys. Rev. Lett. 113, 091102 (2014).

[30] A. Flachi, K. Fukushima, and V. Vitagliano, Phys. Rev. Lett. 114, 181601 (2015).

[31] Y. Hamada, J. M. Pawlowski, and M. Yamada, Phys. Rev. D 103, 106016 (2021).

[32] G. P. de Brito, A. Eichhorn, and M. Schiffer, Phys. Lett. B 815, 136128 (2021).

[33] H. Gies and R. Martini, Phys. Rev. D 97, 085017 (2018).

[34] R. Martini, Aspects of critical phenomena in curved space, Ph.D. thesis, Friedrich-Schiller-Universität Jena, Physikalisch-Astronomische Fakultät, Deutschland, 2019.

[35] H. Gies and S. Lippoldt, Phys. Rev. D 87, 104026 (2013).

[36] H. Gies, J. Jaeckel, and C. Wetterich, Phys. Rev. D 69, 105008 (2004).

[37] J. Braun, M. Leonhardt, and M. Pospiech, Phys. Rev. D 96, 076003 (2017).
[38] J. Braun, M. Leonhardt, and M. Pospiech, Phys. Rev. D 97, 076010 (2018).

[39] J. Braun, M. Leonhardt, and M. Pospiech, Phys. Rev. D 101, 036004 (2020).

[40] H. Gies and S. Lippoldt, Phys. Rev. D 89, 064040 (2014).

[41] S.-B. Liao, Phys. Rev. D 53, 2020 (1996).

[42] S.-B. Liao, Phys. Rev. D 56, 5008 (1997).

[43] R. Camporesi, Commun. Math. Phys. 148, 283 (1992).

[44] D. Vassilevich, Phys. Rep. 388, 279 (2003).

[45] S. Weinberg, in Erice Subnucl. Phys.1976:1 (1976), p. 1.

[46] S. Weinberg, in General Relativity: An Einstein Centenary Survey (1980), pp. 790-831.

[47] M. Reuter, Phys. Rev. D 57, 971 (1998).

[48] D. Dou and R. Percacci, Classical Quantum Gravity 15, 3449 (1998).

[49] M. Niedermaier and M. Reuter, Living Rev. Relativity 9, 5 (2006).

[50] D. F. Litim, Phil. Trans. R. Soc. A 369, 2759 (2011).

[51] M. Reuter and F. Saueressig, New J. Phys. 14, 055022 (2012).

[52] R. Percacci, An Introduction to Covariant Quantum Gravity and Asymptotic Safety (World Scientific, Singapore, 2017).

[53] A. D. Pereira, in Progress and Visions in Quantum Theory in View of Gravity: Bridging foundations of physics and mathematics (2019) [arxiv:1904.07042].

[54] M. Reuter and F. Saueressig, Quantum Gravity and the Functional Renormalization Group: The Road towards Asymptotic Safety (Cambridge University Press, Cambridge, England, 2019).

[55] A. Bonanno, A. Eichhorn, H. Gies, J. M. Pawlowski, R. Percacci, M. Reuter, F. Saueressig, and G. P. Vacca, Front. Phys. 8, 269 (2020).

[56] N. Dupuis, L. Canet, A. Eichhorn, W. Metzner, J. M. Pawlowski, M. Tissier, and N. Wschebor, Phys. Rep. 910, 1 (2021).

[57] M. Reichert, Proc. Sci. 005 (2020) 384.

[58] J. M. Pawlowski and M. Reichert, arXiv:2007.10353.

[59] R. Percacci and D. Perini, Phys. Rev. D 67, 081503 (2003).

[60] A. Codello, R. Percacci, and C. Rahmede, Ann. Phys. (Amsterdam) 324, 414 (2009).

[61] P. Donà and R. Percacci, Phys. Rev. D 87, 045002 (2013).

[62] J. Meibohm, J. M. Pawlowski, and M. Reichert, Phys. Rev. D 93, 084035 (2016).

[63] N. Christiansen, D. F. Litim, J. M. Pawlowski, and M. Reichert, Phys. Rev. D 97, 106012 (2018).

[64] Y. Hamada and M. Yamada, J. High Energy Phys. 08 (2017) 070.

[65] A. Eichhorn and A. Held, Phys. Rev. D 96, 086025 (2017).

[66] G. P. De Brito, A. Eichhorn, and A. D. Pereira, J. High Energy Phys. 09 (2019) 100.

[67] B. Brger, J. M. Pawlowski, M. Reichert, and B.-J. Schaefer, arXiv:1912.01624.

[68] A. Eichhorn and M. Pauly, Phys. Rev. D 103, 026006 (2021).

[69] O. Lauscher and M. Reuter, Phys. Rev. D 66, 025026 (2002).

[70] A. Codello and R. Percacci, Phys. Rev. Lett. 97, 221301 (2006).

[71] A. Codello, R. Percacci, and C. Rahmede, Int. J. Mod. Phys. A 23, 143 (2008). 
[72] D. Benedetti, P. F. Machado, and F. Saueressig, Mod. Phys. Lett. A 24, 2233 (2009).

[73] K. Falls, D. F. Litim, K. Nikolakopoulos, and C. Rahmede, arXiv:1301.4191.

[74] K. Falls, D. F. Litim, K. Nikolakopoulos, and C. Rahmede, Phys. Rev. D 93, 104022 (2016).

[75] H. Gies, B. Knorr, S. Lippoldt, and F. Saueressig, Phys. Rev. Lett. 116, 211302 (2016).

[76] T. Denz, J. M. Pawlowski, and M. Reichert, Eur. Phys. J. C 78, 336 (2018).

[77] N. Christiansen, arXiv:1612.06223.

[78] K. G. Falls, C. S. King, D. F. Litim, K. Nikolakopoulos, and C. Rahmede, Phys. Rev. D 97, 086006 (2018).

[79] A. Eichhorn, P. Labus, J. M. Pawlowski, and M. Reichert, SciPost Phys. 5, 031 (2018).

[80] K. G. Falls, D. F. Litim, and J. Schröder, Phys. Rev. D 99, 126015 (2019).

[81] K. Falls, N. Ohta, and R. Percacci, Phys. Lett. B 810, 135773 (2020).

[82] G. Gubitosi, R. Ooijer, C. Ripken, and F. Saueressig, J. Cosmol. Astropart. Phys. 12 (2018) 004.

[83] A. Eichhorn, Front. Astron. Space Sci. 5, 47 (2019).

[84] J. Biemans, A. Platania, and F. Saueressig, J. High Energy Phys. 05 (2017) 093.

[85] I. L. Buchbinder, S. D. Odintsov, and I. L. Shapiro, Effective Action in Quantum Gravity (IOP, Bristol, 1992).

[86] I. L. Shapiro, P. M. Teixeira, and A. Wipf, Eur. Phys. J. C 75, 262 (2015).

[87] B. S. Merzlikin, I. L. Shapiro, A. Wipf, and O. Zanusso, Phys. Rev. D 96, 125007 (2017).
[88] A. Bonanno and M. Reuter, Phys. Lett. B 527, 9 (2002).

[89] A. Bonanno and M. Reuter, Phys. Rev. D 65, 043508 (2002).

[90] B. Guberina, R. Horvat, and H. Stefancic, Phys. Rev. D 67, 083001 (2003).

[91] M. Reuter and F. Saueressig, J. Cosmol. Astropart. Phys. 09 (2005) 012.

[92] A. Bonanno and A. Platania, Phys. Lett. B 750, 638 (2015).

[93] A. Bonanno and F. Saueressig, C.R. Phys. 18, 254 (2017).

[94] A. Bonanno, A. Platania, and F. Saueressig, Phys. Lett. B 784, 229 (2018).

[95] A. Platania, Universe 5, 189 (2019).

[96] A. Platania, Front. Phys. 8, 188 (2020).

[97] A. Babic, B. Guberina, R. Horvat, and H. Stefancic, Phys. Rev. D 71, 124041 (2005).

[98] B. Koch, P. Rioseco, and C. Contreras, Phys. Rev. D 91, 025009 (2015).

[99] F. Bezrukov, J. Rubio, and M. Shaposhnikov, Phys. Rev. D 92, 083512 (2015).

[100] D. Oriti and H. Pfeiffer, Phys. Rev. D 66, 124010 (2002).

[101] E. Bianchi, M. Han, C. Rovelli, W. Wieland, E. Magliaro, and C. Perini, Classical Quantum Gravity 30, 235023 (2013).

[102] S. Steinhaus, Phys. Rev. D 92, 064007 (2015).

[103] L. Glaser, Classical Quantum Gravity 35, 084001 (2018).

[104] J. Ambjørn, Z. Drogosz, J. Gizbert-Studnicki, A. Grlich, J. Jurkiewicz, and D. Németh, arXiv:2103.00198. 